PROCEEDINGS OF THE

AMERICAN MATHEMATICAL SOCIETY

Volume 127, Number 10, Pages 2943-2952

S 0002-9939(99)05031-5

Article electronically published on April 28, 1999

\title{
ON THE MULTIPLICITY OF EIGENVALUES OF A VECTORIAL STURM-LIOUVILLE DIFFERENTIAL EQUATION AND SOME RELATED SPECTRAL PROBLEMS
}

\author{
CHAO-LIANG SHEN AND CHUNG-TSUN SHIEH
}

(Communicated by Hal L. Smith)

\begin{abstract}
We prove that under certain conditions, a vectorial SturmLiouville differential equation of dimension $n \geq 2$ can only possess finitely many eigenvalues which have multiplicity $n$. For the case $n=2$, we find a sufficient condition on the potential function $Q(x)$, and a bound $m_{Q}$ depending on $Q(x)$, such that the eigenvalues of the equation with index exceeding $m_{Q}$ are all simple. These results are applied to find some sufficient conditions which imply that the spectra of two potential equations, or two string equations, have finitely many elements in common, and an estimate of the number of elements in the intersection of two spectra is provided.
\end{abstract}

\section{INTRODUCTION}

It was known a long time ago that if $q(x)$ is a nice real-valued function on $0 \leq x \leq 1$, and $\rho(x)$ is a nice positive function on $0 \leq x \leq 1$, then the eigenvalues of the potential equation with potential function $q(x)$,

$$
y^{\prime \prime}(x)+(\lambda-q(x)) y(x)=0, y(0)=y(1)=0,
$$

and the eigenvalues of the string equation with density function $\rho(x)$,

$$
y^{\prime \prime}(x)+\mu \rho(x) y(x)=0, y(0)=y(1)=0,
$$

are simple, i.e., of multiplicity 1 , and the $k^{\text {th }}$ eigenfunction has exactly $k-1$ signchanged zeros in the interval $0<x<1$. But for the following $n$-dimensional vectorial Sturm-Liouville differential equation

$$
\vec{z}^{\prime \prime}(x)+(\lambda I-Q(x)) \vec{z}(x)=\overrightarrow{0}, \vec{z}(0)=\vec{z}(1)=\overrightarrow{0},
$$

where $n \geq 2, I$ is the $n$-by- $n$ identity matrix, $Q(x)$ is a continuous $n$-by- $n$ real symmetric matrix-valued function defined on $0 \leq x \leq 1$, and $\vec{z}(x)$ is an $R^{n}$-valued function, the multiplicity of the eigenvalues and properties of the eigenfunctions are not well-understood. One purpose of this paper is to investigate the multiplicity problem of the equation (3) for the case when $Q(x)$ is a Jacobi matrix-valued function, i.e., $Q(x)=\left[q_{i j}(x)\right]$, where $q_{i i}(x)=p_{i}(x), i=1,2,, \ldots, n, q_{j, j+1}(x)=$ $q_{j+1, j}(x)=-r_{j}(x), j=1, \ldots ., n-1$, and $q_{l m}(x)=0$ if $|l-m| \geq 2$. We shall denote

Received by the editors December 29, 1997.

1991 Mathematics Subject Classification. Primary 34A30, 34B24, 34B25, 34L05.

Key words and phrases. Vectorial Sturm-Liouville differential equations, eigenvalues, multiplicity, spectrum, potential equations, string equations, Bessel functions.

(C)1999 American Mathematical Society 
this $Q(x)$ by $J\left[p_{1}(x), \ldots, p_{n}(x) ; r_{1}(x), \ldots, r_{n-1}(x)\right]$, where $p_{i}(x)$ and $r_{j}(x), 1 \leq i \leq n$, $1 \leq j \leq n-1$, are assumed to be continuous functions. In section two, using the theory of matrix differential equations (see [2]), we prove the following theorem among others:

Theorem 2.3. Suppose $Q(x)=J\left[p_{1}(x), \ldots, p_{n}(x) ; r_{1}(x), \ldots, r_{n-1}(x)\right], n \geq 2$. If

$$
\int_{0}^{1} r_{1}(x) d x \neq 0
$$

then, except finitely many eigenvalues, the multiplicity of the eigenvalues of (3) are at most $n-1$.

The case $n=2$ in Theorem 2.3 ( also see Theorem 2.2) is more interesting for application. Let $\sigma(q)$ denote the spectrum of the potential equation (1) with potential function $q(x)$, and let $\sigma_{s}(\rho)$ denote the spectrum of the string equation (2) with density function $\rho(x)$. In section three, as applications of Theorem 2.3, we prove the following results.

Theorem 3.1. Suppose $q_{1}(x)$ and $q_{2}(x)$ are two real-valued continuous functions defined on $0 \leq x \leq 1$. If

$$
\int_{0}^{1} q_{1}(x) d x \neq \int_{0}^{1} q_{2}(x) d x
$$

then $\sigma\left(q_{1}\right)$ and $\sigma\left(q_{2}\right)$ only have finitely many elements in common.

Theorem 3.2. Suppose $\rho_{1}(x)$ and $\rho_{2}(x)$ are two positive $C^{2}$-functions defined on $0 \leq x \leq 1$. If

$$
\int_{0}^{1} \rho_{1}{ }^{1 / 2}(x) d x=\int_{0}^{1} \rho_{2}{ }^{1 / 2}(x) d x
$$

and

$$
\int_{0}^{1}\left(\rho_{1}^{-1 / 4}\right)(x) \frac{d^{2}}{d x^{2}}\left(\rho_{1}^{-1 / 4}\right)(x) d x \neq \int_{0}^{1}\left(\rho_{2}^{-1 / 4}\right)(x) \frac{d^{2}}{d x^{2}}\left(\rho_{2}^{-1 / 4}\right)(x) d x,
$$

then $\sigma_{s}\left(\rho_{1}\right)$ and $\sigma_{s}\left(\rho_{2}\right)$ only have finitely many elements in common.

Finally, in section four, for a two-by-two potential function $Q(x)$ satisfying the condition of Theorem 2.2, we study the problem of finding a number $m_{Q}$ such that for $n \geq m_{Q}$ the $n^{t h}$ eigenvalue of (3) is simple. Suppose $Q(x)=$ $J\left[p_{1}(x), p_{2}(x) ; r_{1}(x)\right], \int_{0}^{1} r_{1}(x) d x \neq 0$. We find two constants $Q_{*}$ and $\Lambda_{*}$, which are expressed in terms of $p_{1}(x), p_{2}(x)$, and $r_{1}(x)$; these two numbers play important roles for studying the above mentioned problem. To be more precise, we have

Theorem 4.1. If $\lambda_{n}$ is an eigenvalue of (3) which satisfies the conditions

$$
\sqrt{\lambda_{n}} \geq 2 Q_{*}, \quad \lambda_{n}>\Lambda_{*},
$$

then $\lambda_{n}$ is a simple eigenvalue.

Using Theorem 4.1, an estimate of $m_{Q}$ is found (see Theorem 4.2), and the result is applied to find an upper bound for the number of elements in the intersection of the spectra of two potential equations (see Theorem 4.3). 


\section{The Multiplicity OF EIGENVAlues OF A VECTORIAL STURM-LiOUVILlE EQUATION}

To study the eigenvalue problem (3) we consider the following matrix differential equation:

$$
Y^{\prime \prime}(x)+(\lambda I-Q(x)) Y(x)=0, Y(0)=0, Y^{\prime}(0)=I,
$$

where $Y(x)$ is an $n$-by-n matrix-valued function. We use $Y(x ; \lambda)$ to denote the solution of the initial value problem (4). Then $\lambda_{*}$ is an eigenvalue of (3) if and only if $Y\left(1 ; \lambda_{*}\right)$ is a singular matrix. The multiplicity of $\lambda_{*}$ as an eigenvalue of $(3)$ is equal to the dimension of the null space of $Y\left(1 ; \lambda_{*}\right)$. It is known (see $[7$, pp 304-305]) that (3) has a countably infinite number of real eigenvalues which are bounded from below. Counting the multiplicity of eigenvalues, we arrange all eigenvalues of (3) as an ascending sequence $\lambda_{1} \leq \lambda_{2} \leq \ldots \leq \lambda_{m} \leq \ldots$. The multiplicity of an eigenvalue $\lambda_{*}$ of $(3)$ is at most $n$, and the multiplicity of $\lambda_{*}$ is $n$ if and only if $Y\left(1 ; \lambda_{*}\right)$ is the zero matrix. The following asymptotic formula of $Y(x ; \lambda)$ ( see $[1,(1.2 .2)]$ ) shall be used later: for $\lambda>0$,

$$
Y(x ; \lambda)=\frac{\sin \sqrt{\lambda} x}{\sqrt{\lambda}} I+O\left(\frac{1}{\lambda}\right), \lambda \rightarrow \infty .
$$

To study the multiplicity of eigenvalues of (3) for

$$
Q(x)=J\left[p_{1}(x), \ldots, p_{n}(x) ; r_{1}(x), \ldots r_{n-1}(x)\right],
$$

first we treat the case $n=2$.

Lemma 2.1. If $Q(x)=J\left[p_{1}(x), p_{2}(x) ; r_{1}(x)\right]$, and $\lambda_{*}$ is an eigenvalue of $(3)$ which has multiplicity two, let $y_{1}(x)=\operatorname{col}\left(y_{11}(x), y_{21}(x)\right)$ and $y_{2}(x)=\operatorname{col}\left(y_{12}(x), y_{22}(x)\right)$ be two solutions of (3) corresponding to $\lambda_{*}$. Then we have the following identity:

$$
\int_{0}^{1} r_{1}(x)\left\{y_{11}(x) y_{22}(x)-y_{12}(x) y_{21}(x)\right\} d x=0 \text {. }
$$

Proof. Note that $y_{i j}(x), i, j=1,2$, satisfy the following two relations:

$$
\begin{aligned}
& y_{11}^{\prime \prime}(x)+\left\{\lambda_{*}-p_{1}(x)\right\} y_{11}(x)+r_{1}(x) y_{21}(x)=0, \\
& y_{12}^{\prime \prime}(x)+\left\{\lambda_{*}-p_{1}(x)\right\} y_{12}(x)+r_{1}(x) y_{22}(x)=0 .
\end{aligned}
$$

By (7) and (8) we have

$$
\begin{aligned}
& \frac{d}{d x}\left\{y_{11}^{\prime}(x) y_{12}(x)-y_{11}(x) y_{12}^{\prime}(x)\right\} \\
& =r_{1}(x)\left\{y_{11}(x) y_{22}(x)-y_{12}(x) y_{21}(x)\right\}
\end{aligned}
$$

Integrating (9) from $x=0$ to $x=1$ and using the boundary conditions $y_{i j}(0)=$ $y_{i j}(1)=0$, we obtain $(6)$.

Using Lemma 2.1 we prove the following result.

Theorem 2.2. If $Q(x)=J\left[p_{1}(x), p_{2}(x) ; r_{1}(x)\right]$, and

$$
\int_{0}^{1} r_{1}(x) d x \neq 0
$$

then, except finitely many eigenvalues, all eigenvalues of (3) are simple. 
Proof. Suppose, on the contrary, there are infinitely many eigenvalues $\lambda_{n_{k}}$ which have multiplicity two. Denote the solution of (4) for $\lambda=\lambda_{n_{k}}$ by

$$
Y\left(x ; \lambda_{n_{k}}\right)=\left[y_{i j}\left(x ; \lambda_{n_{k}}\right)\right]_{i, j=1}^{2} .
$$

Then, by (5), we have

$$
\operatorname{det} Y\left(x ; \lambda_{n_{k}}\right)=\frac{1-\cos \left(2 \lambda_{n_{k}}^{1 / 2} x\right)}{2 \lambda_{n_{k}}}+O\left(\lambda_{n_{k}}^{-3 / 2}\right) .
$$

(6) and (10) imply that

$$
\int_{0}^{1} r_{1}(x)\left\{\frac{1-\cos 2 \lambda_{n_{k}}^{1 / 2} x}{2 \lambda_{n_{k}}}+O\left(\lambda_{n_{k}}^{-3 / 2}\right)\right\} d x=0,
$$

which tells us that we have

$$
\int_{0}^{1} r_{1}(x) d x=\int_{0}^{1} r_{1}(x) \cos \left(2 \lambda_{n_{k}}^{1 / 2} x\right) d x+O\left(\lambda_{n_{k}}^{-1 / 2}\right)
$$

By (11), the Riemann-Lebesgue Lemma tells us that if (3) has infinitely many eigenvalues of multiplicity two, then $\int_{0}^{1} r_{1}(x) d x=0$. Thus if $\int_{0}^{1} r_{1}(x) d x \neq 0$, then all, except finitely many, eigenvalues of (3) are simple.

The result of Theorem 2.2 can be generalized as follows.

Theorem 2.3. Suppose $Q(x)=J\left[p_{1}(x), \ldots, p_{n}(x) ; r_{1}(x), \ldots, r_{n-1}(x)\right], n \geq 2$. If

$$
\int_{0}^{1} r_{1}(x) d x \neq 0
$$

then, except finitely many eigenvalues, the multiplicity of the eigenvalues of (3) are at most $n-1$.

Proof. By Theorem 2.2, the assertion of Theorem 2.3 holds for the case $n=2$. Suppose there exists $Q(x)=J\left[p_{1}(x), \ldots, p_{n}(x) ; r_{1}(x), \ldots, r_{n-1}(x)\right], n \geq 3$, such that the eigenvalue problem (3) has infinitely many eigenvalues $\lambda_{m_{k}}$ of multiplicity $n$. Let $Y\left(x ; \lambda_{m_{k}}\right)=\left[y_{i j}\left(x ; \lambda_{m_{k}}\right)\right]_{i, j=1}^{n}$ denote the corresponding matrix solution of (4). Then following the argument of Lemma 2.1, in particular using (7) and (8), we have (6) for this case. Then, by (5), we also have the identity (11), and hence, by the Riemann-Lebesgue Lemma, we have $\int_{0}^{1} r_{1}(x) d x=0$. Thus if $\int_{0}^{1} r_{1}(x) d x \neq 0$, then, except finitely many eigenvalues, the multiplicity of the eigenvalues of (3) are at most $n-1$.

Theorem 2.4. Suppose $Q(x)=J\left[p_{1}(x), \ldots, p_{n}(x) ; r_{1}(x), \ldots, r_{n-1}(x)\right], n \geq 2$. If at least one $r_{j}(x)$ which is not zero, and $r_{1}(x), \ldots ., r_{n-1}(x)$ never change sign in $0 \leq x \leq 1$, then, except finitely many eigenvalues, the multiplicity of the eigenvalues of (3) are at most $n-1$.

Proof. We shall prove this theorem by mathematical induction. By Theorem 2.2, the assertion of this theorem holds for the case $n=2$. Assume it holds for the case $n=k$, where $k \geq 2$, but there exists a $Q(x)=J\left[p_{1}(x), \ldots, p_{k+1}(x) ; r_{1}(x), \ldots, r_{k}(x)\right]$ such that (3) has infinitely many eigenvalues $\lambda_{m_{t}}$ which have multiplicity $k+1$. Then, by the argument in the proof of Theorem 2.3 and the assumption that $r_{1}(x), \ldots, r_{k}(x)$ never change sign on $0 \leq x \leq 1$, we conclude that $r_{1}(x)=0$. But then for $\tilde{Q}(x)=J\left[p_{2}(x), \ldots, p_{k+1}(x) ; r_{2}(x), \ldots, r_{k}(x)\right]$, the eigenvalues of $(3)$, 
counting multiplicity, consist of the eigenvalues in $\sigma\left(p_{1}\right)$ and the eigenvalues of the eigenvalue problem

$$
\vec{z}^{\prime \prime}(x)+(\mu I-\tilde{Q}(x)) \vec{z}(x)=\overrightarrow{0}, \vec{z}(0)=\vec{z}(1)=\overrightarrow{0},
$$

which implies (12) has infinitely many eigenvalues of multiplicity $k$, a contradiction to the induction assumption. This completes the proof of Theorem 2.4.

\section{The intersection of THE SPECTRA OF TWO POTENTIAL EQUATIONS,} OR TWO STRING EQUATIONS.

In this section we shall apply Theorem 2.2 to study the intersection of the spectra of two potential equations of the form (1), or two string equations of the form (2). Recall that for (1) with potential function $q(x), \sigma(q)$ is used to denote its spectrum, i.e., the set of all eigenvalues of (1); and $\sigma_{s}(\rho)$ is used to denote the spectrum of (2) with density function $\rho(x)$.

Theorem 3.1. Suppose $q_{1}(x)$ and $q_{2}(x)$ are two real-valued continuous functions on $0 \leq x \leq 1$. If

$$
\int_{0}^{1} q_{1}(x) d x \neq \int_{0}^{1} q_{2}(x) d x
$$

then $\sigma\left(q_{1}\right)$ and $\sigma\left(q_{2}\right)$ only have finitely many elements in common.

Proof. Denote

and

$$
\begin{aligned}
& \Lambda(x)=\left[\begin{array}{cc}
q_{1}(x) & 0 \\
0 & q_{2}(x)
\end{array}\right], \\
& U(\theta)=\left[\begin{array}{cc}
\cos \theta & -\sin \theta \\
\sin \theta & \cos \theta
\end{array}\right],
\end{aligned}
$$

$$
\begin{aligned}
Q_{\theta}(x) & =U^{*}(\theta) \Lambda(x) U(\theta) \\
& =J\left[p_{1}(x), p_{2}(x) ; r_{1}(x)\right],
\end{aligned}
$$

where

$$
r_{1}(x)=\left(q_{1}(x)-q_{2}(x)\right) \sin \theta \cos \theta,
$$

$\theta$ is a constant. Then for $Q(x)=Q_{\theta}(x)$, the sequence of eigenvalues, counting multiplicity, of (3), consists of elements of $\sigma\left(q_{1}\right)$ and $\sigma\left(q_{2}\right)$, counting multiplicity. If we choose $\theta$ so that $\sin \theta \cos \theta \neq 0$, then, by (13), $\int_{0}^{1} r_{1}(x) d x \neq 0$. And it follows from Theorem 2.2 that, for $Q(x)=Q_{\theta}(x)$, (3) has only finitely many eigenvalues which have multiplicity two, which implies that $\sigma\left(q_{1}\right) \cap \sigma\left(q_{2}\right)$ is a finite set.

Applying Theorem 3.1 and using the Liouville transformation (see [3]) we obtain the following result.

Theorem 3.2. Suppose $\rho_{1}(x)$ and $\rho_{2}(x)$ are two positive $C^{2}$-functions defined on $0 \leq x \leq 1$. If $\rho_{1}(x)$ and $\rho_{2}(x)$ satisfy the following two conditions,

$$
\begin{gathered}
\int_{0}^{1} \rho_{1}^{1 / 2}(x) d x=\int_{0}^{1} \rho_{2}^{1 / 2}(x) d x \\
\int_{0}^{1}\left(\rho_{1}^{-1 / 4}\right)(x) \frac{d^{2}}{d x^{2}}\left(\rho_{1}^{-1 / 4}\right)(x) d x \neq \int_{0}^{1}\left(\rho_{2}^{-1 / 4}\right)(x) \frac{d^{2}}{d x^{2}}\left(\rho_{2}^{-1 / 4}\right)(x) d x,
\end{gathered}
$$

then $\sigma_{s}\left(\rho_{1}\right)$ and $\sigma_{s}\left(\rho_{2}\right)$ only have finitely many elements in common. 
Proof. Letting

$$
\begin{aligned}
K & =\int_{0}^{1} \rho^{1 / 2}(t) d t, \quad z=K^{-1} \int_{0}^{x} \rho^{1 / 2}(t) d t, \\
u(z) & =\rho^{1 / 4}(x) y(x), \quad \lambda=K^{2} \mu, \\
\theta(z) & =\rho^{1 / 4}(x), \quad q(z)=\theta^{\prime \prime}(z) / \theta(z),
\end{aligned}
$$

the equation (2) is transformed to equation (1) where $y$ and $x$ are replaced by $u$ and $z$, respectively. And, by the method of changing variables, we find that

$$
\int_{0}^{1} q(z) d z=-K^{2} \int_{0}^{1}\left(\rho^{-1 / 4}\right)(x) \frac{d^{2}}{d x^{2}}\left(\rho^{-1 / 4}\right)(x) d x .
$$

Applying (14), (15) and (16), Theorem 3.1 implies our assertion.

As an application of Theorem 3.1, we prove the following result about Bessel functions. For information about Bessel functions, we refer to [4] and [5]. Recall that $J_{n}(x)$ (resp., $Y_{n}(x)$ ) is the Bessel function of the first kind (resp., the second kind) of order $n$.

Corollary 3.3. Suppose $n, k \geq 0, n \neq k, b>1$. Then the functions

$$
J_{n}(s) Y_{n}(s b)-J_{n}(s b) Y_{n}(s)
$$

and

$$
J_{k}(s) Y_{k}(s b)-J_{k}(s b) Y_{k}(s)
$$

have at most finitely many positive zeros in common.

Proof. It is easy to show that if we set

$$
f(x)=\sqrt{x}\left\{J_{n}(s) Y_{n}(s x)-J_{n}(s x) Y_{n}(s)\right\},
$$

then $f(x)$ satisfies the following equation:

$$
f^{\prime \prime}(x)+\left(s^{2}-\frac{n^{2}-1 / 4}{x^{2}}\right) f(x)=0,
$$

with $f(1)=0, f^{\prime}(1) \neq 0$. Thus the square of the positive zeros of the function $J_{n}(s) Y_{n}(s b)-J_{n}(s b) Y_{n}(s)$ are the eigenvalues of the following eigenvalue problem:

$$
y^{\prime \prime}(x)+\left(\lambda-\frac{n^{2}-1 / 4}{x^{2}}\right) y(x)=0, y(1)=y(b)=0 .
$$

Then our assertion is just a consequence of Theorem 3.1.

\section{The Multiplicity of eigenvalues}

\section{OF A TWO-DIMENSIONAL VECTORIAL STURM-LIOUVILLE EQUATION}

Let $Q(x)=J\left[p_{1}(x), p_{2}(x) ; r_{1}(x)\right]$, where $p_{1}(x), p_{2}(x)$ and $r_{1}(x)$ are $C^{1}$-functions on $0 \leq x \leq 1$, and let $\lambda_{1} \leq \lambda_{2} \leq \ldots \leq \lambda_{n} \leq \ldots$ denote the sequence of eigenvalues of (3). If $\int_{0}^{1} r_{1}(x) d x \neq 0$, then, by Theorem 2.2, there exists an index $m_{Q}$ such that for $n \geq m_{Q}$ the eigenvalues $\lambda_{n}$ of (3) are simple. In this section we shall try to find an upper bound estimate of the number $m_{Q}$. This estimate is obviously applicable to the problem of estimating the number of elements in the intersection of the spectra of two potential equations, or two string equations.

Let $A=\left[a_{i j}\right]_{i, j=1}^{2}$ be a two-by-two matrix. Define the maximum norm of $A$ as follows:

$$
\|A\|=\sup \left\{\left|a_{i j}\right|: 1 \leq i, j \leq 2\right\} .
$$


If $A$ and $B$ are two two-by-two matrices, then we have

$$
\|A B\| \leq 2\|A\|\|B\| .
$$

Let $Y(x ; \lambda)$ denote the matrix-valued solution of the equation $(4)$. Then $Y(x ; \lambda)$ satisfies the following integral equation (see [1]):

$$
Y(x ; \lambda)=\frac{\sin \sqrt{\lambda} x}{\sqrt{\lambda}} I+\int_{0}^{x} \frac{\sin \sqrt{\lambda}(x-t)}{\sqrt{\lambda}} Q(t) Y(x ; \lambda) d t .
$$

Denote

$$
Y(x ; \lambda)=\frac{\sin \sqrt{\lambda} x}{\sqrt{\lambda}} I+G(x ; \lambda)
$$

where

$$
\begin{aligned}
G(x, \lambda) & =\left[g_{i j}(x ; \lambda)\right]_{i, j=1}^{2} \\
& =\int_{0}^{x} \frac{\sin \sqrt{\lambda}(x-t)}{\sqrt{\lambda}} Q(t) Y(t ; \lambda) d t .
\end{aligned}
$$

Note that, by (18) and (19), we have

$$
\|Y(x ; \lambda)\| \leq \frac{1}{\sqrt{\lambda}}+2 \int_{0}^{x} \frac{1}{\sqrt{\lambda}}\|Q(t)\|\|Y(t ; \lambda)\| d t,
$$

and, by Gronwall's inequality, (22) tells us that

$$
\|Y(x ; \lambda)\| \leq \frac{1}{\sqrt{\lambda}} \exp \left(\frac{1}{\sqrt{\lambda}} \int_{0}^{x} 2\|Q(t)\| d t\right) .
$$

Then, by (21) and (23), we have, for $1 \leq i, j \leq 2$,

$$
\begin{aligned}
\left|g_{i j}(x ; \lambda)\right| & \leq \frac{1}{\sqrt{\lambda}}\left\{\exp \left(\frac{1}{\sqrt{\lambda}} \int_{0}^{x} 2\|Q(t)\| d t\right)-1\right\} \\
& \leq \frac{1}{\sqrt{\lambda}}\left\{\exp \left(\frac{1}{\sqrt{\lambda}} \int_{0}^{1} 2\|Q(t)\| d t\right)-1\right\} .
\end{aligned}
$$

Thus if

$$
\sqrt{\lambda} \geq \int_{0}^{1} 2\|Q(t)\| d t
$$

then we have, for $1 \leq i, j \leq 2,0 \leq x \leq 1$,

$$
\left|g_{i j}(x ; \lambda)\right| \leq \frac{2 e}{\lambda} \int_{0}^{1}\|Q(t)\| d t .
$$

Using (21) and (20),

$$
\begin{aligned}
\operatorname{det} Y(x ; \lambda) & =\frac{\sin ^{2} \sqrt{\lambda} x}{\lambda}+\frac{\sin \sqrt{\lambda} x}{\sqrt{\lambda}} \operatorname{Trace} G(x ; \lambda)+\operatorname{det} G(x ; \lambda) \\
& =\frac{1-\cos 2 \sqrt{\lambda} x}{2 \lambda}+h(x ; \lambda),
\end{aligned}
$$

where

$$
h(x ; \lambda)=\frac{\sin \sqrt{\lambda} x}{\sqrt{\lambda}} \operatorname{Trace} G(x ; \lambda)+\operatorname{det} G(x ; \lambda),
$$


and, by (25), if (24) is satisfied, then we have, for $0 \leq x \leq 1$,

$$
\lambda|h(x ; \lambda)| \leq \frac{8 e^{2}}{\sqrt{\lambda}} Q_{*},
$$

where

$$
Q_{*}=\int_{0}^{1}\|Q(t)\| d t
$$

And by (27), we have

$$
\left|\int_{0}^{1} \lambda r_{1}(x) h(x ; \lambda) d x\right| \leq \frac{8 e^{2} Q_{*}}{\sqrt{\lambda}} \int_{0}^{1}\left|r_{1}(x)\right| d x .
$$

We shall also need the following inequality, which follows from integration by parts:

$$
\left|\int_{0}^{1} r_{1}(x) \cos 2 \sqrt{\lambda} x d x\right| \leq \frac{\left|r_{1}(1)\right|}{2 \sqrt{\lambda}}+\frac{1}{2 \sqrt{\lambda}} \int_{0}^{1}\left|r_{1}^{\prime}(x)\right| d x .
$$

Note that, by (28) and (29), we have

$$
\begin{aligned}
& \left|\int_{0}^{1} r_{1}(x) \cos 2 \sqrt{\lambda} x d x-2 \lambda \int_{0}^{1} r_{1}(x) h(x ; \lambda) d x\right| \\
& \quad \leq \frac{1}{\sqrt{\lambda}}\left\{\frac{\left|r_{1}(1)\right|}{2}+\frac{1}{2} \int_{0}^{1}\left|r_{1}^{\prime}(x)\right| d x+16 e^{2} Q_{*} \int_{0}^{1}\left|r_{1}(x)\right| d x\right\},
\end{aligned}
$$

if $\lambda$ satisfies inequality (24). Denote

$$
R_{*}=\frac{\left|r_{1}(1)\right|}{2}+\frac{1}{2} \int_{0}^{1}\left|r_{1}^{\prime}(x)\right| d x+16 e^{2} Q_{*} \int_{0}^{1}\left|r_{1}(x)\right| d x .
$$

Now we are ready to prove the following result.

Theorem 4.1. Suppose $Q(x)=J\left[p_{1}(x), p_{2}(x) ; r_{1}(x)\right]$, where $p_{1}(x), p_{2}(x)$ and $r_{1}(x)$ are $C^{1}$-functions on $0 \leq x \leq 1$, and $\int_{0}^{1} r_{1}(x) d x \neq 0$. Let $\Lambda_{*}$ be the positive number defined by

$$
\sqrt{\Lambda_{*}}=\frac{R_{*}}{\left|\int_{0}^{1} r_{1}(x)\right|}
$$

If $\lambda_{n}$ is an eigenvalue of (3) satisfying the following conditions,

$$
\begin{gathered}
\sqrt{\lambda_{n}} \geq 2 Q_{*}, \\
\lambda_{n}>\Lambda_{*},
\end{gathered}
$$

then $\lambda_{n}$ is a simple eigenvalue.

Proof. Suppose $\lambda_{n}$ satisfies conditions (31) and (32), but the multiplicity of $\lambda_{n}$ is two. Then, by Lemma 2.1,

$$
\int_{0}^{1} r_{1}(x) \operatorname{det} Y(x ; \lambda) d x=0
$$

and by (26), we have

$$
\int_{0}^{1} r_{1}(x) d x=\int_{0}^{1} r_{1}(x) \cos 2 \sqrt{\lambda_{n}} x d x-2 \lambda_{n} \int_{0}^{1} r_{1}(x) h\left(x ; \lambda_{n}\right) d x .
$$


But then (30), (31), (32) and (33) imply that

$$
\left|\int_{0}^{1} r_{1}(x) d x\right|<\left|\int_{0}^{1} r_{1}(x) d x\right|
$$

which is absurd.

Note that if $s_{1}(x) \leq s_{2}(x)$ are the characteristic values of the matrix $Q(x)$, then for any scalar-valued functions $f_{1}(x)$ and $f_{2}(x)$ we have

$$
\begin{gathered}
p_{1}(x) f_{1}^{2}(x)-2 r_{1}(x) f_{1}(x) f_{2}(x)+p_{2}(x) f_{2}^{2}(x) \\
\geq s_{1}(x)\left[f_{1}^{2}(x)+f_{2}^{2}(x)\right] .
\end{gathered}
$$

By the maximum-minimum principle ( see $[6$, Chapter VI] ) we have

$$
\lambda_{n}[Q] \geq \lambda_{n}\left[Q_{1}\right]
$$

where $\lambda_{n}[Q]$ is the $n^{\text {th }}$ eigenvalue of $(3)$, and $Q_{1}(x)=s_{1}(x) I, I$ is the two-by-two identity matrix. Since each eigenvalue of (3), with $Q_{1}(x)$ replacing $Q(x)$, is of multiplicity two, we have

$$
\lambda_{n}[Q] \geq\left[\frac{n+1}{2}\right]^{2} \pi^{2}+s_{1, \min },
$$

where $s_{1, \text { min }}$ is the minimum value of $s_{1}(x)$ in $0 \leq x \leq 1$, and $[y]$ is the Gauss symbol of $y$. By (34), Theorem 4.1 implies the following result.

Theorem 4.2. Suppose $Q(x)=J\left[p_{1}(x), p_{2}(x) ; r_{1}(x)\right]$, where $p_{1}(x), p_{2}(x)$ and $r_{1}(x)$ are $C^{1}$-functions on $0 \leq x \leq 1, \int_{0}^{1} r_{1}(x) d x \neq 0$. Let $s_{1}(x)$ be the smaller characteristic value of the matrix $Q(x)$, and let $m_{Q}$ be the smallest positive integer which satisfies the following conditions:

$$
\begin{gathered}
{\left[\frac{m_{Q}}{2}\right]^{2} \pi^{2}+s_{1, \min } \geq 4 Q_{*}{ }^{2},} \\
{\left[\frac{m_{Q}}{2}\right]^{2} \pi^{2}+s_{1, \min }>\Lambda_{*} .}
\end{gathered}
$$

Then the $n^{\text {th }}$ eigenvalues of (3) is simple if $n \geq m_{Q}$.

By Theorem 4.2 and the proof of Theorem 3.1 we also have the following result. We shall use the notation $a \vee b$ to denote the maximum of two real numbers $a$ and $b$. For $q_{1}(x)$ and $q_{2}(x)$, two continuous functions on $0 \leq x \leq 1, \int_{0}^{1}\left(q_{1}(x)-q_{2}(x)\right) d x \neq$ 0 , we shall also use the following abbreviation:

$$
\begin{aligned}
T_{*} & =\left|q_{1}(1)-q_{2}(1)\right|+\int_{0}^{1}\left|q_{1}^{\prime}(x)-q_{2}^{\prime}(x)\right| d x \\
& +16 e^{2} \int_{0}^{1}\left\{\left(\left|q_{1}(x)+q_{2}(x)\right|\right) \vee\left(\left|q_{1}(x)-q_{2}(x)\right|\right)\right\} d x \int_{0}^{1}\left|q_{1}(x)-q_{2}(x)\right| d x, \\
\sqrt{\tilde{\Lambda}_{*}} & =\frac{T_{*}}{2\left|\int_{0}^{1}\left(q_{1}(x)-q_{2}(x)\right) d x\right|} .
\end{aligned}
$$

Theorem 4.3. Suppose $q_{1}(x)$ and $q_{2}(x)$ are $C^{1}$-functions on $0 \leq x \leq 1$, with $\int_{0}^{1}\left(q_{1}(x)-q_{2}(x)\right) d x \neq 0$. Let $s_{*}=\min \left\{q_{1, \min }, q_{2, \min }\right\}$, and let $n_{q_{1}, q_{2}}$ be the 
smallest positive integer which satisfies the following conditions:

$$
\begin{gathered}
{\left[n_{q_{1}, q_{2}}\right]^{2} \pi^{2}+s_{*} \geq\left\{\int_{0}^{1}\left(\left|q_{1}(x)+q_{2}(x)\right|\right) \vee\left(\left|q_{1}(x)-q_{2}(x)\right|\right) d x\right\}^{2},} \\
{\left[n_{q_{1}, q_{2}}\right]^{2} \pi^{2}+s_{*}>\tilde{\Lambda}_{*} .}
\end{gathered}
$$

Then $\sigma\left(q_{1}\right)$ and $\sigma\left(q_{2}\right)$ have at most $n_{q_{1}, q_{2}}$ elements in common.

Proof. In the proof of Theorem 3.1 we choose $\theta=\pi / 4$. Then

$$
\begin{gathered}
Q_{\pi / 4}(x)=\left[\begin{array}{ll}
\frac{1}{2}\left(q_{1}(x)+q_{2}(x)\right) & \frac{1}{2}\left(q_{1}(x)-q_{2}(x)\right) \\
\frac{1}{2}\left(q_{1}(x)-q_{2}(x)\right) & \frac{1}{2}\left(q_{1}(x)+q_{2}(x)\right)
\end{array}\right], \\
r_{1}(x)=-\frac{1}{2}\left(q_{1}(x)-q_{2}(x)\right), \\
\int_{0}^{1}\left\|Q_{\pi / 4}(s)\right\| d s=\frac{1}{2} \int_{0}^{1}\left(\left|q_{1}(x)+q_{2}(x)\right|\right) \vee\left(\left|q_{1}(x)-q_{2}(x)\right|\right) d x,
\end{gathered}
$$

and $\tilde{\Lambda}_{*}$ is the same as the number $\Lambda_{*}$ defined in Theorem 4.1. Suppose, on the contrary, $\sigma\left(q_{1}\right)$ and $\sigma\left(q_{2}\right)$ have at least $n_{q_{1}, q_{2}}+1$ elements in common. Since the sequence of eigenvalues of (3) with $Q(x)=Q_{\pi / 4}(x)$ consists of elements of $\sigma\left(q_{1}\right)$ and $\sigma\left(q_{2}\right)$ counting multiplicity, there exists an index $n_{*}, n_{*} \geq 2 n_{q_{1}, q_{2}}+2$, such that $\lambda_{n_{*}}\left[Q_{\pi / 4}\right]$ is not simple. But, by (34),

$$
\lambda_{n_{*}}\left[Q_{\pi / 4}\right]>\left(n_{q_{1}, q_{2}}\right)^{2} \pi^{2}+s_{*} ;
$$

hence, by (37) and (38), $\lambda_{n_{*}}\left[Q_{\pi / 4}\right]$ satisfies the conditions of Theorem 4.1, which implies that $\lambda_{n_{*}}\left[Q_{\pi / 4}\right]$ is simple, a contradiction. Hence $\sigma\left(q_{1}\right)$ and $\sigma\left(q_{2}\right)$ have at most $n_{q_{1}, q_{2}}$ elements in common.

\section{REFERENCES}

[1] Z. S. Agranovich and V. A. Marchenko, The Inverse Problem of Scattering Theory, Gordon and Breach, New York, 1963. MR 28:5696

[2] F. V. Atkinson, Discrete and Continuous Boundary Problems, Academic Press, New York, 1964. MR 31:416

[3] E. Hille, Lectures on Ordinary Differential Equations, Addison-Wesley, Massachusetts, 1969. MR 40:2939

[4] G. P. Tolstov, Fourier Series, Dover, New York, 1976. MR 54:13429

[5] G. N. Watson, A Treatise on the Theory of Bessel Functions, Cambridge University Press, Cambridge, 1980. MR 96i:33010

[6] R. Courant and D. Hilbert, Methods of Mathematical Physics, Vol. I, Interscience, New York, 1953. MR 16:426a

[7] I. C. Gohberg and M. G. Krein, Theory an Applications of Volterra Operators in Hilbert Space, Translations of Mathematical Monographs, Vol. 24, AMS, 1970. MR 41:9041

Department of Mathematics, National Tsing Hua University, Hsinchu, Taiwan 30043, Republic of China

E-mail address: shen@math.nthu.edu.tw

Current address, Chung-Tsun Shieh: Department of Mathematics, Fu Jen Catholic University, Hsinchuang, Taipei, Taiwan 24205, Republic of China

E-mail address: ctshieh@math.fju.edu.tw 